\title{
Corona citizens' science project-repeated surveys of the Irish response to COVID-19 and subsequent lockdown and restrictive measures
}

\author{
Akke Vellinga $^{1}\left(\mathbb{D} \cdot\right.$ Marc Mellotte $^{2} \cdot$ P J Mealy $^{2} \cdot$ Anthony Staines $^{3}\left(\mathbb{D} \cdot\right.$ Paul O' $^{\prime}$ Connor $^{1}\left(\mathbb{D} \cdot\right.$ Galina Brychkova $^{4}(\mathbb{D})$ \\ Mathieu d'Aquin ${ }^{2}$ (I)
}

Received: 5 January 2021 / Accepted: 26 February 2021 / Published online: 24 March 2021

(c) Royal Academy of Medicine in Ireland 2021

\begin{abstract}
Background Worldwide, many people have been affected by COVID-19, a novel respiratory illness, caused by a new type of coronavirus SARS-CoV2. The COVID-19 outbreak is considered a pandemic and has created a number of challenges for the general population, patients, and healthcare professionals. Lockdowns have been implemented to slow down the spread of the virus with the expectation that these restrictions will limit the number of cases, and hence the number of hospitalizations and ICU admissions. However, these restrictions, and in particular lockdowns, impact on the life of everyone living in Ireland. Aim To record how the COVID-19 pandemic and subsequent restrictive measures impacted on people's activities, work, schooling, and childcare.

Methods The Corona Citizens' Science Project was set up as a population-wide survey. A questionnaire was designed, and the survey was first launched on the 8th of April 2020. An overview of results was released in the press days later. Data was collected in four waves: April 8, April 22, May 6, and June 17, 2020. Each wave had core questions allowing to compare each wave, and wave-specific questions, to understand current impact of changing measures.

Results Over four waves, 152,259 responses were collected. The mean age of respondents was 47 with about $10 \%$ over the age of 65 . Around $75 \%$ were female and $85 \%$ had a higher degree.

Nearly $70 \%$ of the respondents were in employment, and around $13 \%$ were retired. Up to $20 \%$ of the respondents were essential workers, and $10 \%$ of respondents indicated they were in receipt of the COVID-19 pandemic unemployment payment.

Around $10 \%$ of the people who responded were living alone. The number of people talked to the previous day was on average 2.3 in the first survey; during the lockdown, this went up over time, and in the last survey, the mean was 3.9. The percentage of respondents who did not talk to anyone the previous day decreased from 40 to $22 \%$ over the waves.

In the first wave, about $6 \%$ of respondents reported having had flu-like symptoms in the last 14 days, which declined to $3.3 \%$, $2.5 \%$, and $2.0 \%$ in waves 2,3 , and 4 respectively. Similarly, over the four waves, the respondents who indicated that someone they lived with had flu-like symptoms declined from 17 to $12 \%, 9 \%$, and $11 \%$. Throughout the four waves, nearly one third of people reported one or more underlying conditions.

Conclusions As a result of the COVID-19 pandemic, a number of restrictive measures, in particular lockdown, were implemented in Ireland to protect populations and healthcare systems. To record some of the major impacts on society, we launched a Corona Citizens Science Project, with the aim to support decision-making. This report provides detail of its findings.
\end{abstract}

Keywords Corona crisis $\cdot$ COVID-19 $\cdot$ National survey $\cdot$ SARS-CoV2

\section{Introduction}

Worldwide, many people have been affected by COVID-19, a novel respiratory illness, caused by a new type of coronavirus SARS-CoV2. The COVID-19 outbreak was declared a

Akke Vellinga

akke.vellinga@ nuigalway.ie

Extended author information available on the last page of the article pandemic on March 12, 2020 and created a number of challenges for the general population, patients, and healthcare professionals. Restrictions were implemented to slow down the spread of the virus with the expectation that these restrictions will limit the number of cases, and hence the number of hospitalizations and ICU admissions. However, these restrictions also impacted on the life of everyone living in Ireland. 
From the 12th of March to the 18th of May, Ireland implemented "lockdown," which resulted in the closure of all schools and cultural institutions; advice against large gatherings (from the 12th of March) and all non-essential businesses, shops, and venues closed (from the 24th of March). On the 27th of March, this was extended to the advice to stay at home (and work from home). Subsequently, further restrictions and easing of the measures were announced (see overview). Since full lockdown, wearing of face coverings in the community and $2 \mathrm{~m}$ social distancing were implemented (Table 1).

\section{Corona citizens' science project-surveys}

To get a sense of the impact of restrictions on daily life, a survey was organized, based on the survey implemented by the University of Antwerp (https://www.uantwerpen.be/ en/Projects/Corona-study/). The team at the University of Antwerp initiated a weekly national survey on the 17th of March and shared their template and experience.

At the end of March, a website with the survey (Qualtrics) was set up to access and share the survey (nuigalway. ie/Corona-study). The website provided a short introduction
Table 1 Overview of lockdown measures in Ireland from March to October 2020

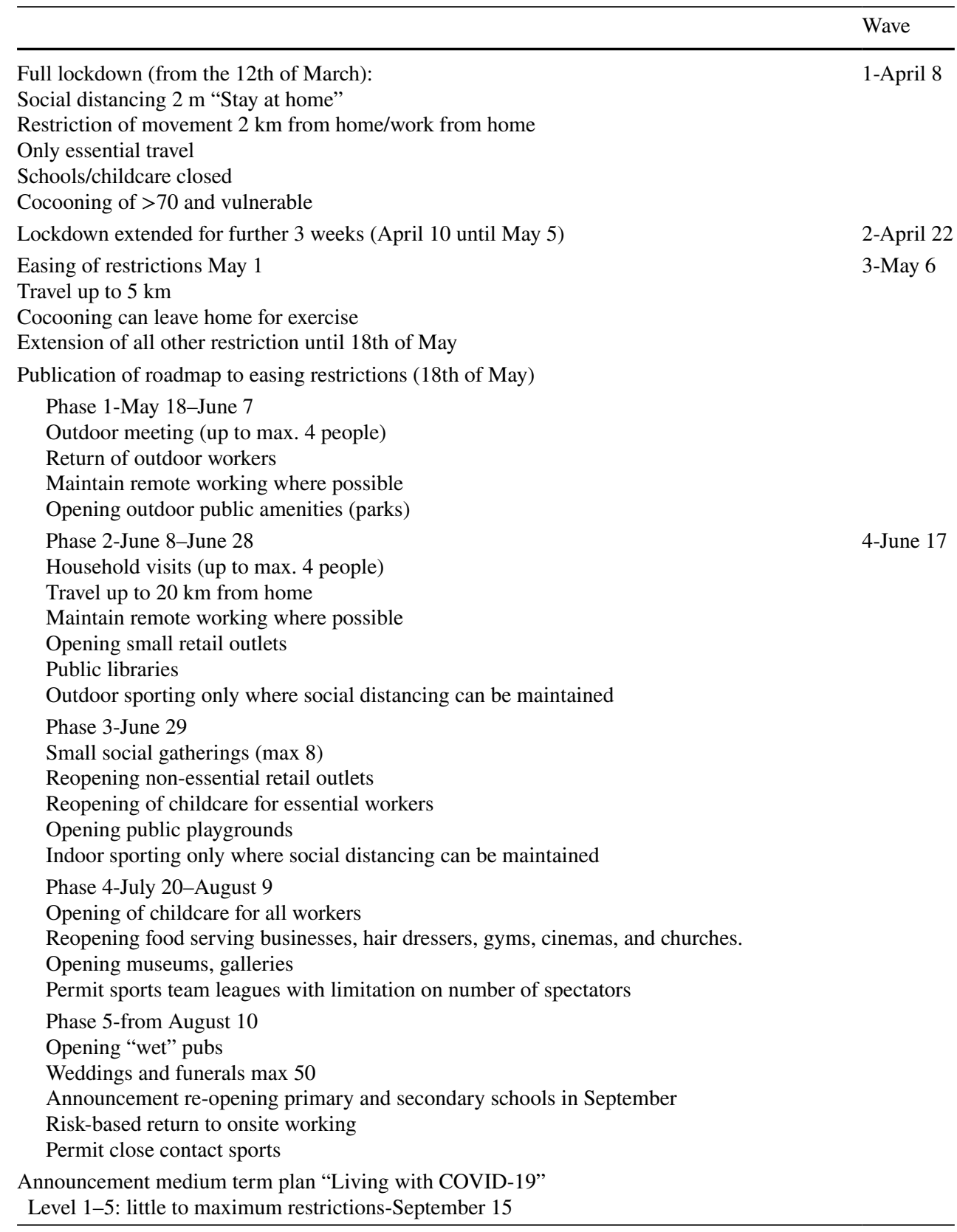


Table 1 (continued)

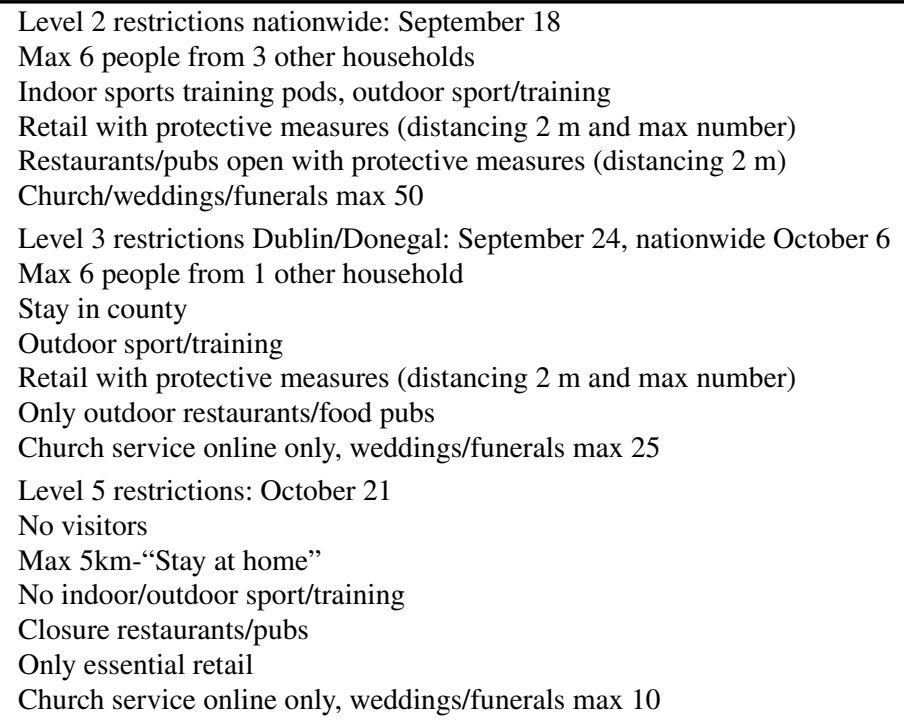

and a link to the survey. The first page of the survey was the consent page, and after indicating consent, the respondent could continue to answering the questions. The survey was voluntary, and no identifiable information was recorded. The survey was open for a 24-h period to allow fast reporting of the results. At the end of this period, all response data was extracted and analyzed. Overview and basic descriptive calculations were performed to write a press release which was issued a day later.

On the 8th of April, the first survey was launched and distributed through personal networks, mainly via Whatsapp and other social media sharing. Repeat surveys (22nd of April, 6th of May, and 17th of June) were launched each time with a section of demographic baseline questions and additional questions informed by the stage of the lockdown/ restrictions.

After the first call on the 8th of April (wave 1), more than 100,000 people responded. Media interest was generated, and the next survey was adapted and made available for launch on the 22nd of April (wave 2), which could be filled during over the next $24 \mathrm{~h}$ before closing again. About 35,000 people responded to the second wave. Subsequent surveys were launched on the 6th of May (8682) and 22nd of June (2936).

\section{Questionnaire}

The first survey covered the following areas:

- Demographic data: geographic area, age, sex, occupation, children, internet connection, receipt of COVID-
19 unemployment payment, number of people in household.

- Home schooling, childcare arrangements, and working from home.

- Presence/absence of COVID-19 symptoms, underlying health conditions, management of testing/symptoms by healthcare professionals.

- Attitudes and adherence to the government's recommendations

- Activities, relational tensions, well-being, and mental health (based on HSE questionnaire).

Additional questions in the second survey (22nd of April)

- Doing their Leaving certificate (final year secondary national exam), cocooning (government advice for elderly people to stay inside the house and not meet people)

- Delayed treatment or check-ups for other illnesses

- Attitudes and adherence to updated government recommendations and lifting of restrictions

- A comment box was included

By the third survey (6th of May), the governments' plan to lift restrictions was just launched, and people were asked if they understood the plan and if they would adhere to this. In addition, more detail on the delayed treatments and the people's views of the planned introduction of a tracing app were requested.

For the fourth wave, to encourage people's involvement, a call was launched through the press to collect questions 
from the public to be included in the next survey. Based on the responses, the questionnaire was reorganized (with demographic data being moved to the end of the survey), and questions on breaking of restrictions, change in drinking/eating/exercise, views of reopening of schools, previous severe flu episodes (pre-COVID), attitudes to vaccines (flu and COVID-19 when available) and antibody testing, and the impact of COVID-19 on sexual relations were included. The fourth wave including these new questions was launched June 17.

A website https://corona-study.nuigalway.ie/was set up to visualize more detail of the survey and allow people to have a more detailed look at the data by selecting certain waves or population groups.

\section{Results}

\section{Demographic profile (Table 2)}

Over the four waves, female respondents were in the majority with $75 \%$ (Fig. 1). The mean and median age was 47, and this was the same for males and females (Fig. 2). Most responses came from Dublin (up to 30\%) and Galway

Table 2 Overview of demographic information of the respondents over the four waves of the Corona Citizens Science Project. Table provides the number of responses, mean age, and number of people talked to in previous days, and percentages in each category

\begin{tabular}{|c|c|c|c|c|c|}
\hline & Wave 1 & Wave 2 & Wave 3 & Wave 4 & All \\
\hline Respondents & 105,926 & 24,715 & 8682 & 2936 & 152,259 \\
\hline Participation in Wave 1 & 105,926 & 12,605 & 3485 & 1233 & \\
\hline Wave 2 & & & 3033 & 1098 & \\
\hline Wave 3 & & & & 784 & \\
\hline Age (mean) & 47.0 & 46.7 & 50.9 & 48.0 & 47.2 \\
\hline$<35$ & 18.5 & 19.6 & 13.3 & 15.0 & 18.4 \\
\hline $35-65$ & 71.7 & 70.8 & 72.5 & 74.9 & 71.6 \\
\hline \multirow[t]{2}{*}{$>65$} & 9.7 & 9.6 & 14.2 & 10.1 & 10.0 \\
\hline & $\%$ & $\%$ & $\%$ & $\%$ & $\%$ \\
\hline$\%$ female & 76.2 & 70.6 & 76.9 & 77.7 & 75.0 \\
\hline \multicolumn{6}{|l|}{$\%$ by area } \\
\hline Dublin & 27.8 & 30.2 & 29.4 & 20.2 & 28.3 \\
\hline Galway & 12.3 & 13.8 & 15.9 & 28.0 & 13.1 \\
\hline Cork & 6.5 & 7.4 & 7.8 & 7.1 & 6.8 \\
\hline Limerick & 2.5 & 2.5 & 1.2 & 2.4 & 2.4 \\
\hline Other counties & 51.0 & 46.1 & 45.6 & 42.3 & 49.4 \\
\hline$\%$ Higher degree (beyond secondary school) & 86.2 & 87.7 & 86.6 & 90.4 & 86.7 \\
\hline$\%$ Living alone & 10.4 & 10.4 & 11.6 & 12.0 & 10.5 \\
\hline$\%$ Cocooning & & 14.3 & 13.2 & 7.2 & 13.7 \\
\hline \multicolumn{6}{|l|}{ \% Employment } \\
\hline Employed & 68.8 & 69.3 & 62.7 & 68.0 & 68.5 \\
\hline Unemployed & 1.7 & 2.1 & 1.8 & 2.6 & 1.8 \\
\hline Home maker & 7.7 & 7.1 & 7.4 & 7.7 & 7.5 \\
\hline Student & 4.1 & 4.1 & 4.1 & 4.5 & 4.1 \\
\hline Retired & 12.8 & 12.9 & 19.3 & 12.6 & 13.2 \\
\hline Other & 4.9 & 4.5 & 4.7 & 4.5 & 4.8 \\
\hline$\%$ Essential worker (of people who are in employment) & 19.4 & 21.3 & 24.7 & 21.3 & 20.1 \\
\hline \multirow[t]{2}{*}{$\%$ COVID pandemic unemployment payment } & 10.3 & 10.7 & 9.8 & 7.8 & 10.3 \\
\hline & $N$ & $N$ & $N$ & $N$ & $N$ \\
\hline \multirow[t]{2}{*}{ Mean number of people talked to previous day } & 2.3 & 2.5 & 2.7 & 3.8 & 2.4 \\
\hline & $\%$ & $\%$ & $\%$ & $\%$ & $\%$ \\
\hline$\%$ of respondents who did not talk to anybody the previous day & 39.7 & 37.7 & 32.1 & 22.2 & 38.4 \\
\hline $\begin{array}{l}\% \text { of respondents who lived alone and did not talk to anybody the } \\
\text { previous day }\end{array}$ & 31.6 & 31.7 & 25.6 & 37.5 & 31.4 \\
\hline
\end{tabular}




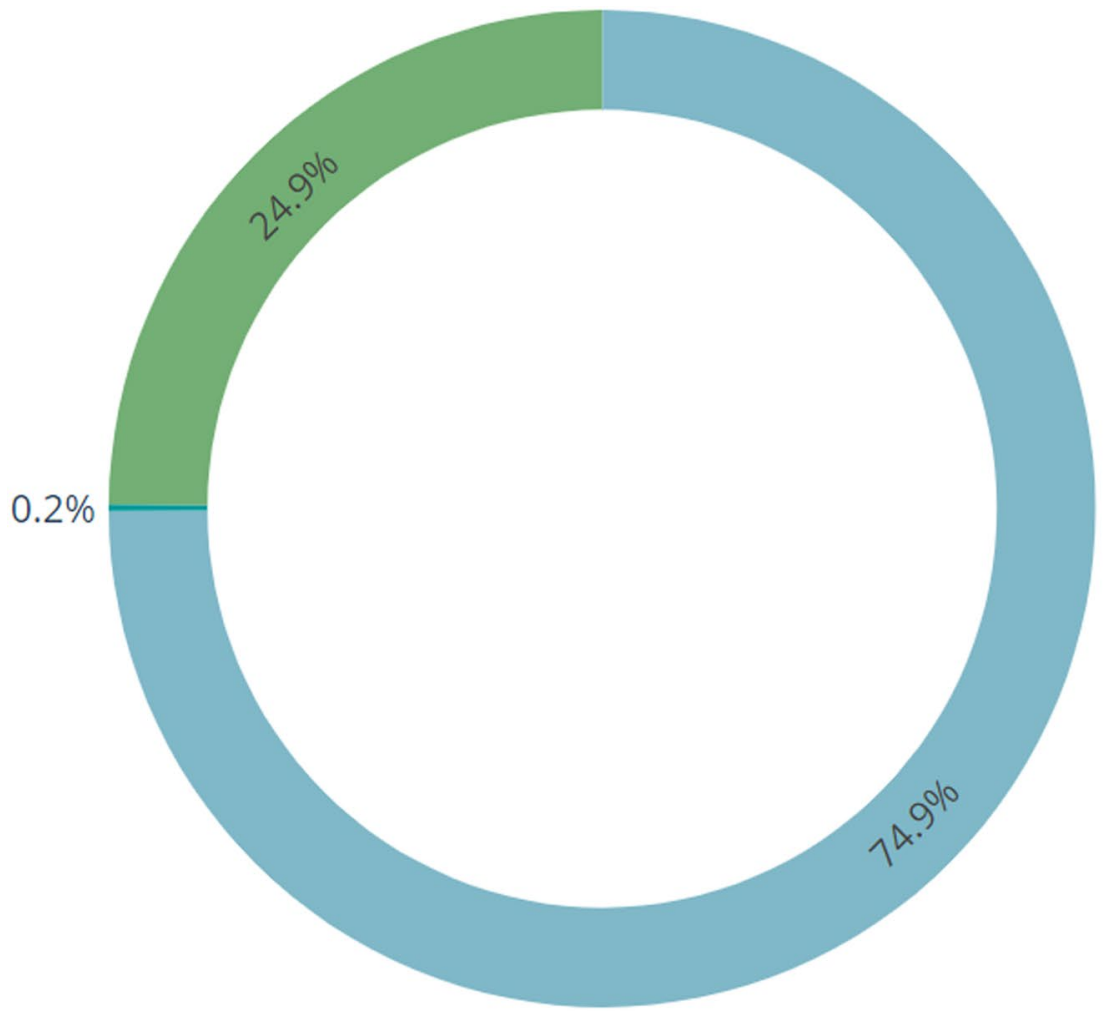

\section{Female \\ Male \\ - Other}

Fig. 1 Female respondents

(13\%) and then Cork (6/7\%) and all other counties at less than $5 \%$ (Fig. 3).

Overall, the level of education was high; generally more than $60 \%$ of the respondents had a university degree.

Nearly $70 \%$ of the respondents were in employment and around $13 \%$ were retired (with the exception of the second survey, where $19 \%$ were retired). Around $20 \%$ of the employed respondents were essential workers, who had to go into work in retail or healthcare. Of the respondents, $10 \%$ indicated they were in receipt of the COVID-19 pandemic unemployment payment.

Around $10 \%$ of the people who responded were living alone. From the second survey on, a question on cocooning was added, and this decreased from $14.3 \%$ in the second to $13.2 \%$ in the third and $7.2 \%$ in the fourth survey, when restrictions were lifted somewhat.

The number of people talked to the previous day was on average 2.3 in the first survey; during the lockdown, this went up over time, and in the last survey, the mean was 3.9. The percentage of respondents who did not talk to anyone the previous day decreased from 40 to $22 \%$ over the waves. In general, older respondents talked on average to slightly less other people face-to-face than younger respondents; under $35 \mathrm{~s}$ met about $2.1,2.2,2.3$, and 3.4, respectively, during each wave; $35-65$ met on average 2.3,
2.6, 2.9, and 4.0, respectively; while over 65 s met $1.5,1.8$, 2.2 , and 3.2 , respectively.

\section{Flu-like symptoms (Table 3)}

In the first wave, about $6 \%$ of respondents reported having had flu-like symptoms in the last 14 days. Symptoms indicative of COVID-19 reported were fever (19\%), sore throat $(54 \%)$, cough $(57 \%)$, runny nose $(42 \%)$, and/or pain in the chest $(21.3 \%$ ) (Fig. 4. Over the waves, the proportion of respondents indicating they had flu-like symptoms declined, to $3.3 \%, 2.5 \%$, and $2.0 \%$, respectively, and so did their symptoms. The loss of smell or taste question was only included after the first wave when it emerged as an apparent symptom of COVID-19.

Similarly, over the four waves, the respondents who indicated someone they lived with had flu-like symptoms in the previous 2 weeks also declined, from 17 to $12 \%, 9 \%$, and $11 \%$. Throughout the four waves, nearly one third of people reported one or more underlying conditions.

The changing testing criteria and capacity for testing changed over time which was reflected in the numbers of positive tests as well as the number of people waiting for a test or its result. In the first wave, $36 \%$ of respondents with 


\section{Age Distribution of Respondants}

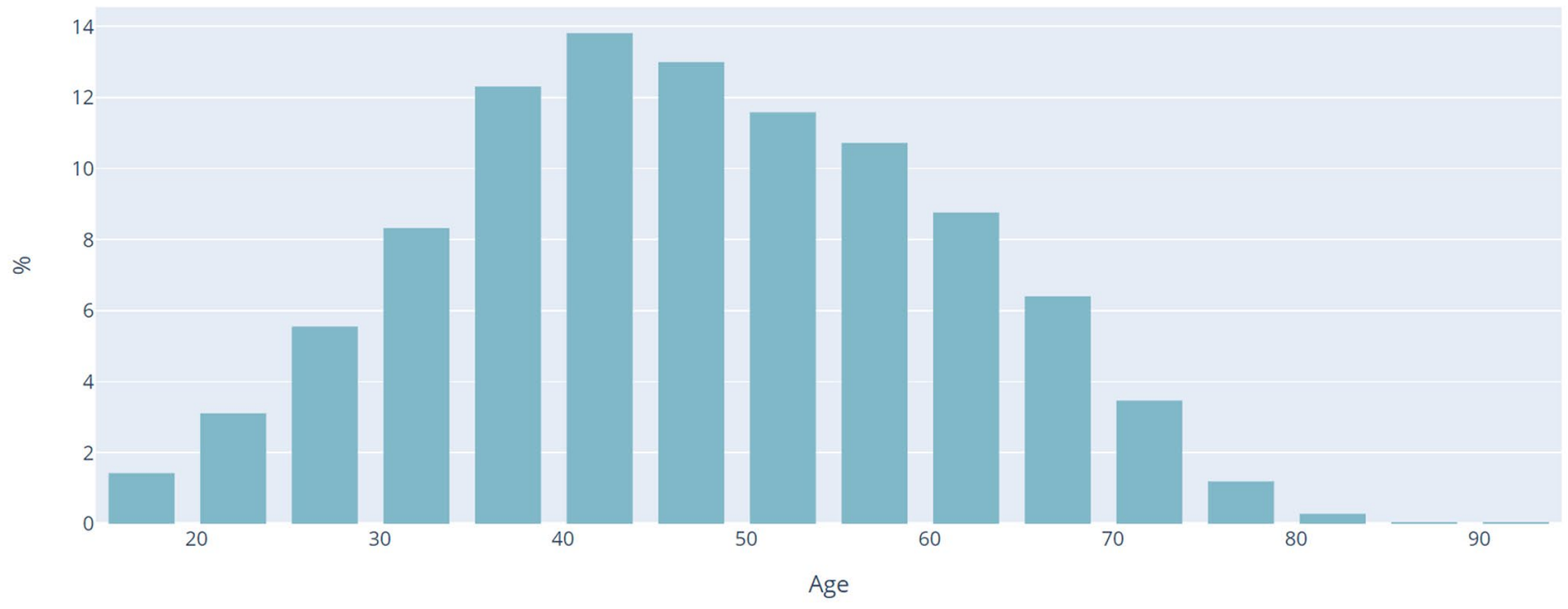

Fig. 2 Mean and median age

flu-like symptoms were referred for testing, and this dipped 2 weeks later (27\%), but remained fairly stable at around a third. Nearly three quarters were either waiting to be tested or waiting for the result of their test in the first wave, which dropped to $22 \%$ in the second, $31 \%$ in the third, and by June, $31 \%$ were waiting. Of people who did receive test results, $10 \%$ were positive in the first wave, going up to $25 \% 2$ weeks later, $13 \%$ in the third wave, and $11 \%$ a month later.

\section{Relationship tensions and anxiety (Tables 4 and 5)}

The question on how the relationship tensions with other adults, children, and/or the partner changed could be answered on a Likert scale from less than usual to much more than usual. Interactions with adults over 70 saw tension that was "more than usual" for $9 \%$, in the first wave, which gradually declined over time. Tensions in the interactions with adults between 18 and 70 were reported for $8.2 \%$ as "much more than usual" which increased slightly to $9 \%$ for the next two waves but declined again for the fourth wave. About $8 \%$ of interaction with children between 12 and 17 were considered to have increased tension, but again, this declined in June. A similar increase in tension was reported with children under the age of 12 . Tensions with partners were "much more than usual" for $7 \%$ in the first wave, increasing to nearly $9 \% 2$ weeks later and $8 \%$ another 2 weeks later; however, this dropped to $7 \%$ in June. In general, women were more likely than men to experience any relationship tensions, within each of the age groups as well as their partner.
In the third and fourth survey, a question on anxiousness since the COVID-19 outbreak was included and asked if respondents were much more, more, same of less anxious, or worried than usual.

About $61 \%$ respondents reported being more or much more anxious in wave 3 which was $57 \%$ in the following wave. Less anxiousness was reported by $8 \%$ in wave 3 and $10 \%$ in wave 4.

If people reported being more or much more anxious, they were asked for the main reasons for this, and more than one answer was allowed. The anxiety was mainly due to worry about catching the virus $(78 \%$ and $70 \%$ in wave 3 and wave 4 , respectively), while $37 \%$ and $35 \%$ also reported to worry about other health problems, $33 \%$ and $34 \%$ about the relaxation of restrictions, and $26 \%$ and $25 \%$ about their finances or their business. Relatively more people worried about working from home in the fourth wave, $31 \%$ (previously $24 \%$ ) and $29 \%$ (previously $24 \%$ ) about their child's schooling.

\section{Wave-specific findings and questions}

\section{Wave 1}

In the first wave, April 8, more detail was asked in relation to the sudden change in working circumstances while children were also at home as schools closed. Of the people who were in employment $6 \%$ used to work from home every day, $19 \%$ only sometimes and $41 \%$ never. When respondents indicated previous working from home, this was only occasionally or just 1 day a week for $80 \%$. The reason respondents did not 


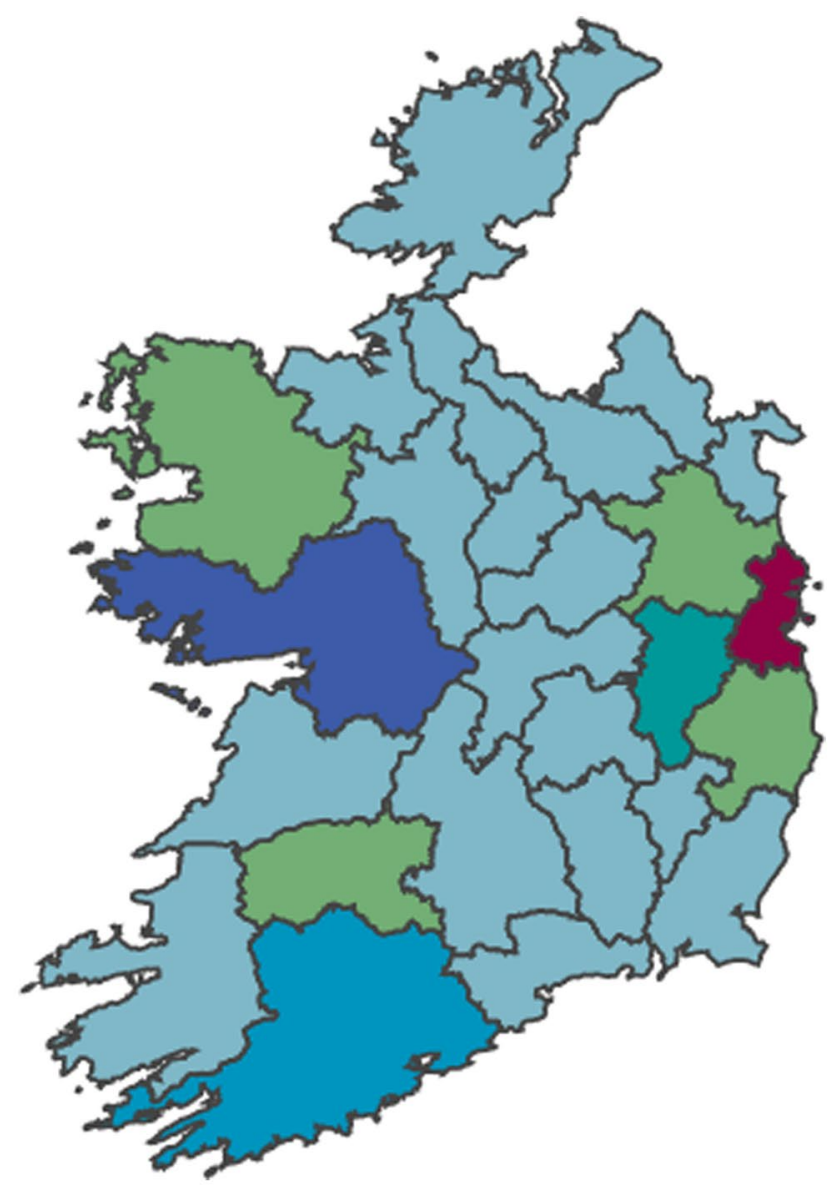

Fig. 3 Most responses from Dublin (up to 30\%) and Galway (13\%) and then Cork $(6 / 7 \%)$ and all other counties at less than 5\%

work from home was usually because their job required face to face contact (44\%), and $17 \%$ indicated that working from home was not allowed in their company.

At the start of lockdown, about $11 \%$ of respondents had children in preschool of whom $89 \%$ were cared for at home, $5 \%$ were with a childminder, $4 \%$ were cared for by family, and $2.5 \%$ were with their grandparents.

About $24 \%$ of respondents had children in primary school. Of these people, $77 \%$ did not encounter any problems with schooling, but some indicated they did not have the necessary resources, such as a laptop or access to computers (3\%), or had insufficient resources because there were not enough laptops/computers to go around (10\%), not enough access to information (5\%), or an unreliable internet connection (4\%).

About $10 \%$ of respondents had a child or children under the age of 15 in secondary school. Most schools tried to continue the curriculum by sending work home (30\%) and $67 \%$ sent home work but also provided additional supports online. For children between 15 and 17, the figures were $31 \%$ and $61 \%$, respectively.
At the moment of the first wave, lockdown was in place for 3 weeks, since the 12th of March with more measures implemented from the 24th of March. The main points were in relation to social distancing of $2 \mathrm{~m}$; isolation (no meeting outside household), ban on travel, and leisure remain within 2-km radius of home and closing of all businesses except for those considered selling essentials.

Most people found the recommendations from the government clear. A score of eight or higher (out of ten) was given by $92 \%$ of the respondents for social distancing, $83 \%$ on isolation recommendations, $80 \%$ for leisure and travel, and $79 \%$ on shopping.

In each case, the commonest value chosen was 10/10, suggesting that the government's recommendations were well understood, by most people. Men, younger people, and people not in formal employment were all likely to assess the clarity of communication slightly less favorably.

The large majority of people report that they adapted their behavior, and that others have done the same. At home, 85\% feel they strongly adapted their behavior ( 9 or 10 on scale), and $66 \%$ find others also strongly adapted. In public places, $75 \%$ feel they strongly adapted their behavior but think only $37 \%$ of the people around them strongly adapted.

\section{Wave 2}

The second survey was launched on the 22nd of April, nearly 1 month into lockdown. As schools were still closed, most children were taken care of at home. Essential workers had other means of childcare, with carers (10\%) and family/ grandparents (15\%), in addition to home care.

Parents of children in primary school ran into obstacles with the school work, such as their child's motivation (54\%), their own work $(40 \%)$, other children in the house $(24 \%)$, and clarity around what was expected (18\%). For secondary school parents, the main obstacles were also motivation (55\%) and clarity (20\%). Overall, $17 \%$ of the parents of school children of any age identified resources as an obstacle.

About $32 \%$, nearly 11,000 people, postponed medical treatment or check-ups. Of these patients, $41 \%$ had preventative routine examinations postponed, $48 \%$ a consultation with the GP, $14 \%$ had a hospital medical examination, and $6 \%$ an operation. More detail was given by about a fifth of the respondents with delayed/cancelled treatments. Dental appointments, blood tests, orthopedic procedures, and follow-up consultations after an operation were most often mentioned. Of particular interest are parents reporting postponement of vaccination and pre- and postnatal check-ups, while fertility treatment has also been halted.

At full lockdown, between the 12th of March and the 22nd of April, the main restrictions included the 
Table 3 Overview of symptoms reported by respondents with flu-like symptoms, mean duration of these symptoms, the percentage of people around the respondents with flu-like symptoms. In addition, testing of the respondents with symptoms and any underlying conditions of the respondents is reported by wave

\begin{tabular}{|c|c|c|c|c|c|}
\hline & Wave 1 & Wave 2 & Wave 3 & Wave 4 & All \\
\hline \multirow[t]{2}{*}{ Respondents } & 105,926 & 24,715 & 8,682 & 2,936 & 152,259 \\
\hline & $\%$ & $\%$ & $\%$ & $\%$ & $\%$ \\
\hline Flu like symptoms & 5.7 & 3.3 & 2.5 & 2.0 & 4.9 \\
\hline \multicolumn{6}{|l|}{ IF YES-symptoms: } \\
\hline Fever & 19.1 & 19.8 & 12.0 & 6.9 & 18.9 \\
\hline Sore throat & 53.9 & 52.1 & 47.5 & 37.9 & 53.3 \\
\hline Shortness of breath & 30.0 & 28.9 & 29.5 & 22.4 & 29.8 \\
\hline Cough & 57.3 & 51.2 & 41.5 & 34.5 & 55.7 \\
\hline Pain in chest & 21.3 & 21.4 & 12.0 & 17.2 & 21.0 \\
\hline Loss of smell or taste & & 19.2 & 16.6 & 17.2 & 18.8 \\
\hline Runny nose & 42.3 & 36.9 & 31.8 & 39.7 & 41.2 \\
\hline \multirow[t]{2}{*}{ Sore eyes } & 21.3 & 22.3 & 20.2 & 20.7 & 21.4 \\
\hline & $N$ & $N$ & $N$ & $N$ & $N$ \\
\hline \multirow[t]{2}{*}{ Mean duration of symptoms (days) } & 10.7 & 10.3 & 9.0 & 7.9 & 10.6 \\
\hline & $\%$ & $\%$ & $\%$ & $\%$ & $\%$ \\
\hline People around you with symptoms & 16.8 & 12.2 & 9.5 & 11.0 & 15.0 \\
\hline$>70$ & 0.8 & 0.6 & 0.7 & & 0.7 \\
\hline $18-70$ & 12.4 & 9.5 & 7.3 & & 11.2 \\
\hline \multirow[t]{2}{*}{$<18$} & 6.3 & 3.2 & 2.1 & & 5.2 \\
\hline & $\%$ & $\%$ & $\%$ & $\%$ & $\%$ \\
\hline \multicolumn{6}{|l|}{ Of people with symptoms } \\
\hline Contacted GP & 53.7 & 42.6 & 50.7 & 29.3 & 51.7 \\
\hline Referred for testing & 36.4 & 27.2 & 30.9 & 32.8 & 34.7 \\
\hline Positive & 9.7 & 24.5 & 13.4 & 10.5 & 11.6 \\
\hline Negative & 18.2 & 41.7 & 50.7 & 68.4 & 22.3 \\
\hline Waiting for test & 35.4 & 4.5 & 11.9 & 5.3 & \\
\hline Waiting for results & 36.6 & 17.2 & 19.4 & 15.8 & 33.6 \\
\hline Test cancelled & & 12.1 & 4.5 & 0 & 1.6 \\
\hline Underlying condition & 26.6 & 27.0 & 28.3 & 28.3 & 26.8 \\
\hline Heart condition & 3.1 & 3.3 & 3.6 & 3.2 & 3.2 \\
\hline Lung condition & 2.2 & 2.1 & 2.3 & 2.6 & 2.1 \\
\hline Diabetes & 2.3 & 2.2 & 2.6 & 2.4 & 2.3 \\
\hline High blood pressure & 10.5 & 10.7 & 11.9 & 10.4 & 10.6 \\
\hline Obesity & 2.8 & 3.0 & 3.2 & 4.1 & 2.9 \\
\hline
\end{tabular}

limitation of movement to $2 \mathrm{~km}$ from the home; working from home; closure of shops, restaurants, and pubs; closure of schools; and limit social contacts to household contacts only. Participants were asked to rank these five different restrictions in order in which they would prefer to see these relaxed. Across all groups (parents, young people, working people), lifting the 2-km limit was ranked first, second was to allow small group meetings; third was to go back to work; fourth was to open schools and childcare again; and in last place to open shops, restaurants, and pubs again.

Percentage of people who gave a ranking for each restriction (first to be lifted to last (ranked 5th) to be lifted) (Table 6).

\section{Wave 3}

A total of 8700 people responded to the third wave of whom $35 \%$ participated in the first wave and $40 \%$ in the second.

Childcare arrangements remained similar. Of preschool children, most were taken care of at home, but when looking at differences between non-essential and essential workers, $93 \%$ were taken care of at home compared with $68 \%$ for essential workers. Essential workers had to rely more often on childminders ( $18 \%$, up from $10 \%$ previously), family (12\%), and grandparents (4\%), compared with non-essential workers (respectively 4\%, 3\%, and 1\%).

Of the primary school children, $29 \%$ had daily contact with their school teacher, while $21 \%$ had contact 2-3 times a 


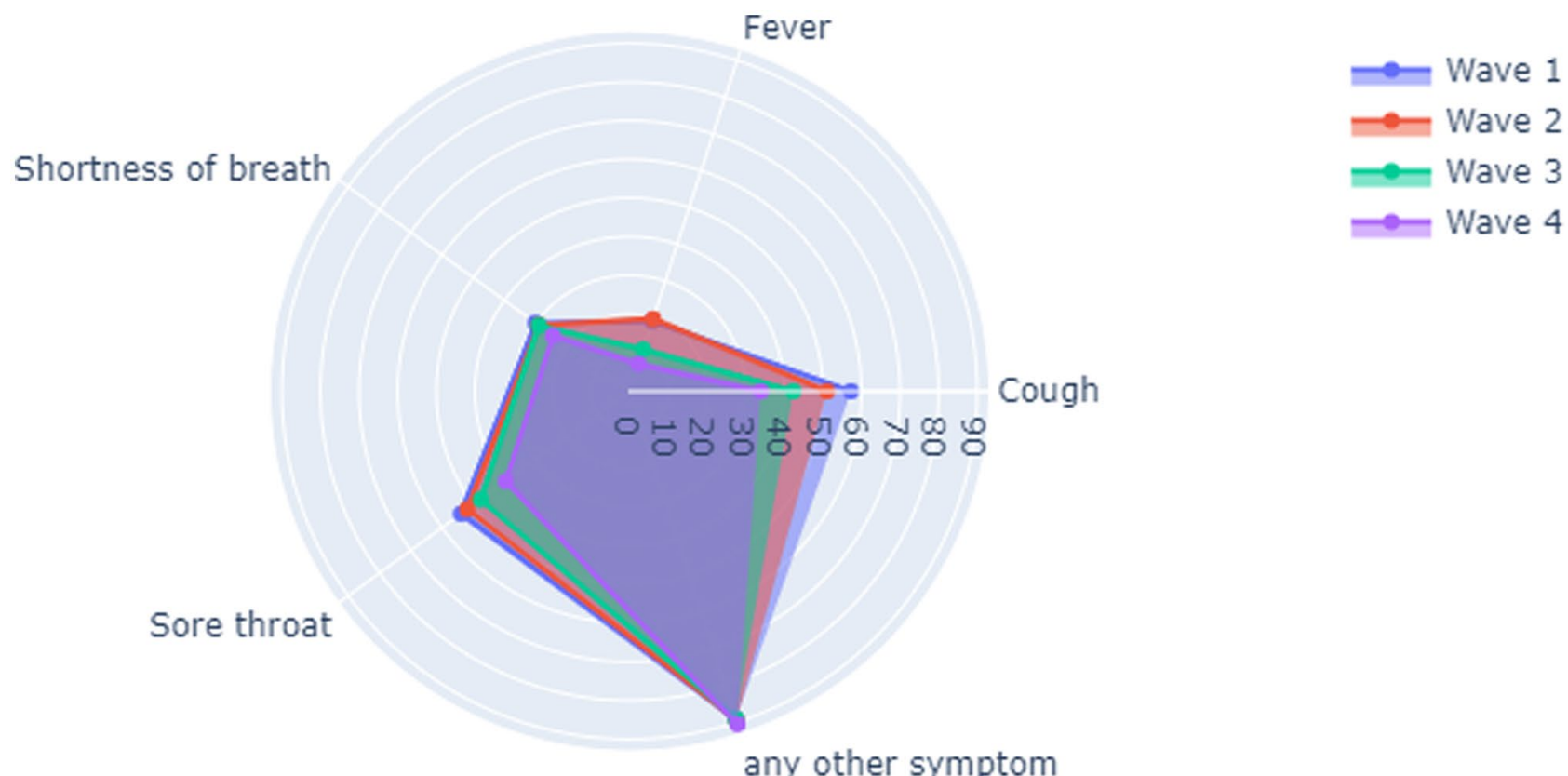

Fig. 4 Symptoms indicative of COVID-19

week and $47 \%$ once or less often each week. For $3 \%$ of children, there was no contact with their primary school teacher.

Parents of secondary school children reported that $64 \%$ of under 15 and $54 \%$ of over 15 year olds had daily contact with their teacher, $25 \%$ and $28 \%$ respectively $2-3$ times a week, and $11 \%$ and $18 \%$ once a week or less often.
The government phased plan received an eight or higher (on a scale of 10) from $72 \%$ of respondents, which was similar to the understanding of which activities were allowed from the first phase (72\%); however, it was less clear when people could go back to work (55\% gave an 8 or higher) or when businesses they needed would open up again (56\%).
Table 4 Response to the question if relationship tensions changed according to the respondents' interaction with other adults, children, and partner for each of the waves

\begin{tabular}{lccccc}
\hline & Wave 1 & Wave 2 & Wave 3 & Wave 4 & All \\
\hline Any relational tensions with & & & & & \\
Adults $>$ 70 & 46,867 & 17,853 & 4,639 & 1,858 & 71,217 \\
& $\%$ & $\%$ & $\%$ & $\%$ & $\%$ \\
A little more than usual & 20.3 & 19.9 & 19.5 & 19.0 & 20.1 \\
Much more than usual & 9.0 & 8.1 & 7.8 & 7.2 & 8.7 \\
Adults 18-70 & 79,241 & 27,661 & 6,914 & 2,472 & 116,288 \\
& $\%$ & $\%$ & $\%$ & $\%$ & $\%$ \\
A little more than usual & 28.7 & 29.5 & 28.1 & 27.7 & 28.8 \\
Much more than usual & 8.2 & 9.0 & 9.0 & 7.0 & 8.4 \\
Children 12-17 & 37,150 & 13,018 & 3,199 & 1,354 & 54,721 \\
& $\%$ & $\%$ & $\%$ & $\%$ & $\%$ \\
A little more than usual & 22.4 & 21.9 & 21.9 & 21.0 & 22.2 \\
Much more than usual & 8.0 & 8.1 & 8.0 & 6.6 & 8.0 \\
Children <12 & 43,929 & 15,027 & 3,324 & 1,467 & 63,747 \\
A little more than usual & $\%$ & $\%$ & $\%$ & $\%$ & $\%$ \\
Much more than usual & 27.1 & 26.2 & 24.9 & 26.9 & 26.8 \\
Partner & 8.0 & 8.1 & 8.0 & 6.6 & 8.0 \\
A little more than usual & 76,014 & 26,313 & 6,632 & 2,277 & 111,236 \\
Much more than usual & $\%$ & $\%$ & $\%$ & $\%$ & $\%$ \\
\hline & 27.3 & 28.3 & 25.7 & 25.6 & 27.4 \\
& 7.4 & 8.5 & 8.0 & 7.2 & 7.7 \\
\hline
\end{tabular}


Table 5 Response to the question in relation to the anxiousness of the respondent. This question was only introduced in wave 3 and repeated in wave 4

\begin{tabular}{lcc}
\hline & Wave 3 & Wave 4 \\
\hline Respondents to this question & 8636 & 2908 \\
& $\%$ & $\%$ \\
Much more anxious or worried than usual & 14.0 & 14.5 \\
More anxious or worried than usual & 47.2 & 42.9 \\
More or less the same as before the outbreak & 30.6 & 32.4 \\
Less anxious or worried(more content) & 8.2 & 10.2 \\
\hline
\end{tabular}

When asked how easily people found it to comply with these restrictions, $78 \%$ gave an eight or higher (ten being very easy to comply) for the $2-\mathrm{km}$ travel restriction, $74 \%$ for working from home, and $78 \%$ gave an eight or higher for keeping social distancing.

A COVID-19 tracking app was planned to be launched on the week after the third wave, and respondents were asked if they would install a tracking app, if this would become available. Overall, $84 \%$ of respondents indicated they would install such an app. Males were less likely to consider this compared with females (15 v 18\%). People who said they would not install such an app rated the governmental phase plan consistently lower, and this was similar to the questions on how easy/difficult it would be to comply with the restrictions.

The postponement of medical treatment was again included in this wave, but more detail was requested. About $31 \%$ of respondents postponed medical treatment or check-ups, similar to the second wave, mainly because the healthcare professional was not seeing any patients (56\%, second wave 55\%), 32\% (second wave 39\%) because they did not want to create an extra burden, and 23\% (second wave $26 \%$ ) were worried about the risk of catching COVID-19.

Of respondents who postponed treatment, $36 \%$ (second wave $48 \%$ ) postponed a consultation with the GP, and $15 \%$ postponed a consultation with another primary care professional. About $14 \%$ had a hospital medical examination postponed (same as second wave) and 7\% (second wave 6\%) an operation. Dental appointments (35\%), check-ups (36\%), counseling $(5 \%)$, and diabetic clinics $(2.4 \%)$ were the main other delayed medical appointments.

\section{Wave 4}

A total of 2620 people responded in the fourth wave of whom $42 \%$ participated in wave $1,37 \%$ in wave 2 , and $27 \%$ in wave 3.

About $21 \%$ ( 1 in 5) of the people recalled a severe flu in the last winter, with symptoms that would subsequently
Table 6 Ranking of the lockdown restrictions according to which the respondent would like to see lifted first to last. Percentage of respondents who favored lifting of each restriction according to rank

\begin{tabular}{lcccrr}
\hline Rank & First & 2nd & 3rd & 4th & \multicolumn{1}{c}{ 5th } \\
\hline Be able to go outside beyond 2 km & 51.1 & 23.8 & 12.1 & 8.2 & 4.8 \\
Small group meetings & 19.1 & 37.6 & 18.1 & 14.6 & 10.5 \\
Go back to work & 17.9 & 19.4 & 33.9 & 20.6 & 8.2 \\
Open schools/childcare & 8.3 & 11.7 & 19.7 & 32.9 & 27.4 \\
Open shops/restaurants/pubs & 3.6 & 7.5 & 16.2 & 23.7 & 49.0 \\
\hline
\end{tabular}

be considered COVID-19. Most of these were reported for December (39\%) and January (27\%), while 21\% reported it in February, 9\% in November, and 5\% in October.

In relation to the flu, 35\% received the flu vaccine in the previous winter (2019) and 57\% intended to get before the winter 2020. If a COVID-19 vaccine would become available, $59 \%$ said they would get it, $32 \%$ maybe, and $8 \%$ would not consider it. If antibody testing would become available, $48 \%$ would do this immediately and $44 \%$ if medically indicated, while $7 \%$ did not want an antibody test.

Sexual relationships were not affected by COVID-19 for most (73\%) of the respondents, but $10 \%$ indicate better and $18 \%$ worse relationships. The negative impact on sexual relationships was especially felt by younger age groups with $42 \%$ of the under 25 age group indicating a negative impact and $29 \%$ of the $25-35$ year olds. Of respondents who smoked (12\%), 46\% indicated they smoked more during lockdown while $18 \%$ smoked less. Half of the respondents indicated they drank alcohol of which $28 \%$ indicated to drink more while $22 \%$ drank less. Exercise increased for $40 \%$ of the people, while $24 \%$ exercised less. In reply to their weight, $36 \%$ of respondents put on weight, while $13 \%$ lost some.

Over the previous months, $25 \%$ of the participants had a tele-consultation with their GP, $81 \%$ for non-COVID related, and $19 \%$ for COVID-related symptoms. Most respondents found it effective (67\%), but 17\% were not sure and 16\% did not find it an effective way of communication.

In relation to lockdown, which was planned to be lifted by the next few weeks, about a third of the respondents did not worry at all about coming out of lockdown and would like to see this happen as soon as possible (31\%); $39 \%$ of respondents were concerned, because of catching COVID-19, $12 \%$ because of infecting others, and $4 \%$ indicated they developed social anxiety. The under 25 age group is much more likely to have indicated such social anxiety $(17 \%)$ while the older age groups are more worried about catching COVID-19 (up to $51 \%$ in the over 65 ).

About half of the respondents felt the government was balancing well between COVID-19 restrictions and other considerations such as other public health, societal, and economic wellbeing ( $48 \%$ of respondents gave an eight or higher on the 
scale of ten); the median mark was 7. Seventeen percent of respondents felt that the government was taking too much risk, but most respondents gave this a five, neither too much nor too little.

About $30 \%$ of the respondents were more careful $(\geq 8)$ about going out and adhering to restrictions, while most respondents were more or less the same (5 on a scale of 10). And if there was a next wave, $85 \%$ indicated they would adhere to restrictions again $(\geq 8)$.

Face masks were worn by $52 \%$ of respondents, more likely to be older. In the under 25 age group, $40 \%$ compared with $75 \%$ in the over 65 age group. If they did not wear a face mask, $73 \%$ indicated they would wear a face mask if this means reducing distancing from 2 to $1 \mathrm{~m}$. Most respondents said they never broke any restrictions $(53 \%, 1373)$, while $44 \%$ occasionally, and $3 \%$ regularly.

About $15 \%$ of respondents were worried about being made redundant, younger age groups more so than older. There were $68 \%$ of respondents with children, and they felt their child(ren) suffered a bit (47\%) and a lot (33\%) due to the lack of social interaction. Overall, $68 \%$ indicated they would send their child to school if schools would reopen.

In relation to college, if they or their child intended to start college in September, most were still going ahead with this plan $(71 \%)$, while $17 \%$ had not decided yet and $6 \%$ intended to defer due to uncertainty.

\section{Discussion}

The Corona Citizens' Science Project was the first national survey performed in Ireland to understand and document the response of the Irish people to COVID-19 and lockdown. Over the course of the project, more than 150,000 respondents submitted answers to the questions.

The research team included questions with the aim to support and guide decision-making at the national level by providing the impact of these measures on the people living in Ireland. Every wave was extensively reported in the national press.

Over four waves, 152,259 responses were collected. The mean age of respondents was 47 with about $10 \%$ over the age of 65 . Around $75 \%$ were female and $85 \%$ had a higher degree. So, even though more than 150,000 responses were collected, the sample was not fully representative. More females than males responded overall, and the level of education was exceptionally high, which is probably a reflection of the use of personal networks of the researchers involved. The impact of the underrepresentation of males and younger age groups is difficult to establish but in particular to the younger age groups, the restrictive measures may have been more challenging.
The lockdown has impacted different population groups differently. With around $10 \%$ of the people who responded were living alone, this group may have suffered particularly from isolation, enhanced by a lack of communication. People learned to communicate and interact differently, and over time, the number of people who did not talk to anyone the previous day decreased from $40 \%$ at the start of April 2020 to 22\% in June 2020.

The impact of lockdown on the spread of the virus could indirectly be seen in the reduction of respondents with flu-like symptoms, from 6\% in April 2020 to 2.0\% in June 2020. Similarly, the number of respondents who reported flu-like symptoms in someone they lived with declined from 17 to $11 \%$.

Various interesting details emerged throughout the waves, showing overall support for the governmentimposed restrictions, nearly $60 \%$ indicated they would get the vaccine if it became available, $84 \%$ would download a smartphone COVID tracker app, and up to $20 \%$ of respondents had a serious flu in the months leading up to the COVID pandemic.

The Corona Citizens' Science Project started as a direct response to the corona crisis and was set up as an unfunded and voluntary project by three researchers at NUI Galway. Subsequently, Dublin City University joined and provided vital press support, and other researchers volunteered to provide statistical and press support. No funding could be obtained to continue this project, and lack of time and resources limited the number of waves. Survey and lockdown fatigue became apparent over the course of 4 waves and after the disappointing results of the fourth wave, a temporary stop was decided. However, considering the enormous success of the project and the continuing spread of the COVID-19 virus with restrictive public health responses, a further wave would have been most informative. Interested researchers are invited to explore the data upon request, and funders interested in supporting another wave should contact the authors.

Acknowledgements We would like to thank Professor Pierre Van Damme and Koen Pepermans for sharing the Corona Survey and providing invaluable support.

We would like to acknowledge the help from the Dublin City Press Officer, Claire Egan and Professor Charles Spillane of the Ryan Institute. Qualtrics with the help of Tim Strik provided the software to set up the survey for free.

Sarah O’Connor, Sana Parveen, Suzanne Kelly, Shashank Bhartiya, and Raziullah Thazathe Peedika provided support in the fourth wave to reach more respondents through social media. Fionnuala Rogers provided help with statistical analyses.

Author contribution AV conceived of the study, obtained ethical approval, designed the first survey, analyzed the data, wrote press releases, and prepared the manuscript. MM set up the website, provided content and technical support, and developed the questionnaires. PJM provided the technical support to set up each questionnaire in 
Qualtrics. AS analyzed the data, wrote press releases, and provided overall support to the project. POC provided support with the analysis of the mental health and wellbeing sections. GB provided further indepth analysis of results from each wave. MDA supervised the project and provided technical support through the Data Science Institute. Both $\mathrm{AV}$ and AS were available for comment after each press release. All the authors were involved in the development of questions for each wave, and the distribution of each wave through personal networks, mainly via Whatsapp and other social media sharing. All the authors contributed to drafts of the manuscript and read the final manuscript.

Funding No funding was received for this study. Qualtrics (qualtrics.com) with the help of Tim Strik provided free access to survey software.

Availability of data and material All data is available on request. More information can be found at https://corona-study.nuigalway.ie/.

\section{Declarations}

Code availability Code can be downloaded from https://github.com/ stainesa/CitizenScience.

Ethics approval Ethical approval was obtained from the Ethical Committee of the Royal College of Surgeons Ireland.

Conflict of interest The authors declare that they have no conflict of interest.

Publisher's Note Springer Nature remains neutral with regard to jurisdictional claims in published maps and institutional affiliations.

\section{Authors and Affiliations}

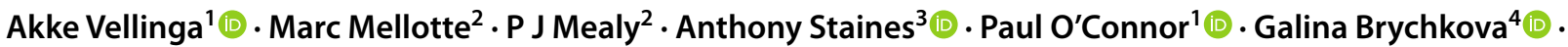 Mathieu d'Aquin² (1)}

Marc Mellotte

Marc.mellotte@nuigalway.ie

P J Mealy

pjmealy@gmail.com

Paul O'Connor

Paul.oconnor@nuigalway.ie

Galina Brychkova

galina.brychkova@nuigalway.ie

Mathieu d'Aquin

Mathieu.daquin@nuigalway.ie
1 Epidemiologist School of Medicine National, University of Ireland, Galway, Ireland

2 Insight Centre for Data Analytics National, University of Ireland, Galway, Ireland

3 Professor of Health Systems School of Nursing, Psychotherapy and Community Health, Dublin City University, Glasnevin, Dublin 9, Dublin, Ireland

4 Ryan Institute National, University of Ireland, Galway, Ireland 